\title{
Stability Behavior of Perforated Lipped Channel Columns
}

\author{
Chungang $\mathrm{WANG}^{1}$, Yu BAI ${ }^{1}$, Qingqing $\mathrm{LIU}^{1}$ and Daqian $\mathrm{ZHAO}{ }^{1}$ \\ ${ }^{1}$ Shenyang Jianzhu University, Shenyang, Liaoning China
}

\begin{abstract}
Taking the cold-formed thin-walled perforated lipped channels with simple edge stiffeners and complex edge stiffeners as research objects. The influence of perforated parameters, such as hole width, hole height, hole shape and hole spacing, on axial compressive bearing capacity and buckling mode of these two types of lipped channels were studied by finite element analysis. It shows that the change of hole width, hole shape and hole spacing may change the buckling mode, but influence little on ultimate bearing capacity within a reasonable perforated range; However, it indicates that the ultimate bearing capacity decreased obviously with the opening height increased. The suggestions on reasonable hole shape and hole size were put forward. Furthermore, combined the direct strength method of lipped perforated channels under axial compression abroad with the relevant specification of our country, the direct strength method of lipped perforated channel columns for our country were put forward also. Then, the methods mentioned above were extended to calculate the ultimate bearing capacity of perforated channels with complex edge stiffeners under axial compression, and the validity of the formulas was verified.
\end{abstract}

\section{Introduction}

Cold formed thin-walled steel is widely used in all kinds of industrial and civil buildings for its flexible cross section form and efficient structural characteristics. In order to facilitate the installation of circuit system and pipeline facilities, holes are usually set up on the web of cold-formed thin-walled steel members in practical engineering application. But the existence of holes can affect the stability and bearing capacity of the members, even change the buckling modes. And the calculation method of bearing capacity becomes more complicated. The current direct strength method (DSM) is no longer suitable for the perforated members. Therefore, it is necessary to carry out the relevant research on cold-formed thin-walled perforated lipped channels under axial load.

For perforated lipped channels and complex lipped channels pin-ended columns, the influence of perforated parameters on bearing capacity and buckling mode has been studied. And combined the direct strength method of perforated common lipped channels under axial compression abroad with the relevant specification of our country, the direct strength method of perforated common lipped channel columns for our country were put forward in this paper. Then the methods mentioned above were extended to calculate the ultimate bearing capacity of

* Corresponding author:ralphsy@163.com 
perforated channels with complex edge stiffeners under axial compression, and the validity of the formulas was verified.

\section{The Influence Factors of Stability Performance by Finite Element Parametric Analysis}

The accuracy of simulating method has been validated by preliminary study [1]. The paper uses the same finite element analysis method to study the influence of hole width, hole height, hole shape and hole spacing on bearing capacity and buckling mode of lipped and complex lipped perforated channels. The reasonable hole shape and hole size are put forward.

There are 50 lipped perforated channels and 58 complex lipped perforated channels. The cross section forms used for analysis are showed in Fig.1. In the specimen label, L and the number then represents effective length; Ja and the number then means the serial number of lipped perforated channels and $\mathrm{Jb}$ means complex lipped channels(Table 1); the letter $\mathrm{b}$ and then means the width of hole; the letter $h$ and then means the height of hole. When there is a single hole in the web, the hole in the specimen length $1 / 2$; there are two holes, the holes in the specimen length $1 / 3$. The hole spacing $l$ means the distance between the center of holes. The web holes sizes parameters are showed in Fig2.

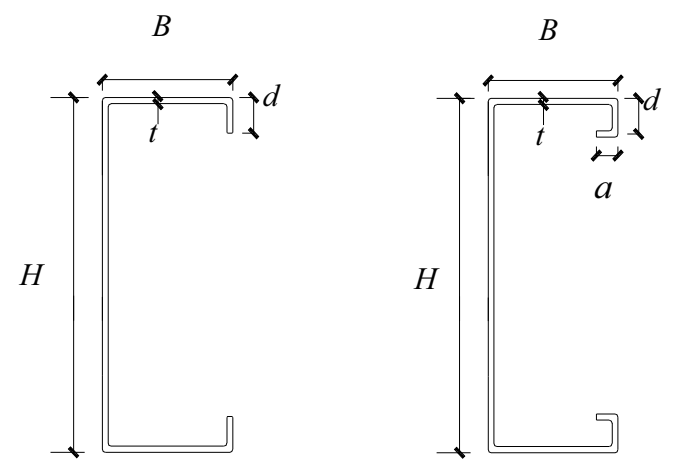

$\begin{array}{lll}\text { (a) lipped channel } & \text { (b) complex lipped channel }\end{array}$

Fig.1 Cross section of specimen

$l$

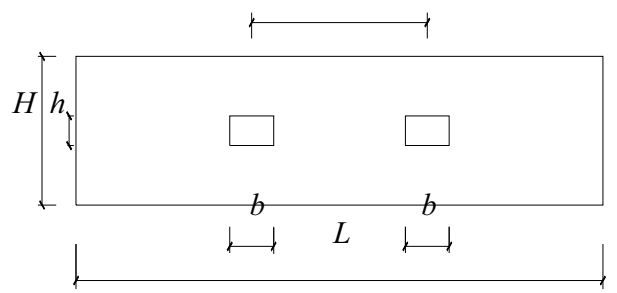

Fig.2 Web holes sizes parameters 
Table1 Cross Section Numbers Of Specimen

\begin{tabular}{|c|c|c|c|c|c|}
\hline Specimen & $H / \mathrm{mm}$ & $B / \mathrm{mm}$ & $d / \mathrm{mm}$ & $a / \mathrm{mm}$ & $t / \mathrm{mm}$ \\
\hline $\mathrm{Ja} 1$ & 200 & 90 & 25 & - & 2 \\
\hline $\mathrm{Ja} 2$ & 200 & 120 & 25 & - & 2 \\
\hline $\mathrm{Ja} 3$ & 200 & 120 & 25 & - & 2.5 \\
\hline $\mathrm{Ja} 4$ & 160 & 70 & 20 & - & 2 \\
\hline $\mathrm{Ja} 5$ & 160 & 90 & 20 & - & 3 \\
\hline $\mathrm{Ja} 6$ & 180 & 70 & 20 & - & 2 \\
\hline $\mathrm{Ja} 7$ & 180 & 90 & 20 & - & 2 \\
\hline $\mathrm{Ja} 8$ & 180 & 120 & 20 & - & 1 \\
\hline $\mathrm{Ja} 9$ & 220 & 120 & 25 & - & 2 \\
\hline $\mathrm{Ja} 10$ & 220 & 150 & 25 & - & 2 \\
\hline $\mathrm{Jb} 1$ & 200 & 90 & 25 & 15 & 2 \\
\hline $\mathrm{Jb} 2$ & 200 & 120 & 25 & 15 & 2 \\
\hline $\mathrm{Jb3}$ & 200 & 120 & 25 & 15 & 2.5 \\
\hline $\mathrm{Jb} 4$ & 200 & 100 & 25 & 15 & 2 \\
\hline $\mathrm{Jb} 5$ & 200 & 180 & 25 & 15 & 2 \\
\hline $\mathrm{Jb} 6$ & 160 & 70 & 20 & 10 & 2 \\
\hline $\mathrm{Jb} 7$ & 160 & 90 & 20 & 10 & 2 \\
\hline $\mathrm{Jb} 8$ & 150 & 150 & 25 & 15 & 2 \\
\hline $\mathrm{Jb} 9$ & 150 & 150 & 25 & 15 & 3 \\
\hline $\mathrm{Jb} 10$ & 120 & 120 & 20 & 10 & 2 \\
\hline $\mathrm{Jb} 11$ & 120 & 120 & 20 & 10 & 3 \\
\hline $\mathrm{Jb} 12$ & 220 & 120 & 25 & 15 & 2 \\
\hline $\mathrm{Jb} 13$ & 250 & 220 & 30 & 20 & 2 \\
\hline $\mathrm{Jb} 14$ & 300 & 280 & 35 & 15 & 2 \\
\hline
\end{tabular}

\subsection{The hole width}

The results of finite element analysis are showed in Table 2. The bearing capacity of specimen decreases with the increase of hole width, but the amplitude is very weak. From Table 2, it's obvious that the influence of the width of hole on the buckling mode of members is very small. It is suggested that the hole width of the cold-formed channel under axial compression is $110 \mathrm{~mm}$.

\subsection{The hole height}

As shown in Table 3. The ultimate bearing capacity of the member is obviously decreased with the increase of the height of the hole and the influence of the hole height on the stability of the bearing capacity is large.

In order to find a more economical and reasonable hole height range, the ratio of bearing capacity of specimen $\left(P_{\mathrm{u}}\right)$ and net section strength carrying capacity $\left(P_{\text {ynet }}\right)$ is used as ordinate and the ratio of the height of hole $(h)$ and the height of web $(H)$ is used as abscissa. $P_{\text {ynet }}=f_{\mathrm{y}} \times A_{\text {net }}$, $f_{\mathrm{y}}$ means yield strength, $A_{\text {net }}$ means net cross-sectional area. The results are showed in Fig3. It is suggested that hole height should be $30-40$ percent of the height of web. 


\subsection{The hole shape}

The bearing capacities of perforated channel under axial compression with different hole shapes are showed in Table 4. It's showed that the influence of hole shape on bearing capacity is small. But considered concentration of stress and opening technology, it's suggested that elliptical hole is better than rectangular hole.

\subsection{The hole spacing}

The ultimate bearing capacity of the axial compression members with different hole spacing is shown in Table 5. The influence of hole spacing on bearing capacity is small. Referred to the relevant codes, the minimum height of specimen is not less than $1200 \mathrm{~mm}$ for members with two holes.

Table2 Bearing Capacity Of Perforated Lipped Channel With Different Hole Width

\begin{tabular}{|c|c|c|c|}
\hline Specimen & $P_{\mathrm{u}} / \mathrm{kN}$ & Specimen & $P_{\mathrm{u}} / \mathrm{kN}$ \\
\hline L700Ja7b40h40 & 165.48 & L700Jb1b70h60 & 175.89 \\
\hline L700Ja7b70h40 & 164.47 & L700Jb1b100h60 & 174.14 \\
\hline L700Ja7b100h40 & 163.07 & L700Jb1b40h80 & 170.48 \\
\hline L700Ja1b40h40 & 169.55 & L700Jb1b70h80 & 168.85 \\
\hline L700Ja1b70h40 & 170.82 & L700Jb1b100h80 & 166.37 \\
\hline L700Ja1b100h40 & 169.95 & L1200Jb7b60h40 & 174.92 \\
\hline L900Ja10b60h60 & 184.45 & L1200Jb7b90h40 & 172.57 \\
\hline L900Ja10b80h60 & 183.34 & L1200Jb7b70h60 & 159.71 \\
\hline L900Ja10b100h60 & 182.84 & L1200Jb7b100h60 & 158.39 \\
\hline L700Jb1b40h40 & 181.46 & L1200Jb1b60h40 & 176.02 \\
\hline L700Jb1b70h40 & 181.59 & L1200Jb1b90h40 & 175.43 \\
\hline L700Jb1b100h40 & 180.29 & L1200Jb1b70h60 & 167.48 \\
\hline L700Jb1b40h60 & 176.57 & L1200Jb1b100h60 & 166.46 \\
\hline
\end{tabular}

Table 3 Bearing Capacity Of Perforated Channel With Different Hole Shapes

\begin{tabular}{|c|c|c|}
\hline Specimen & Shapes & $P_{\mathrm{u}} / \mathrm{kN}$ \\
\hline L700Ja1b100h40 & Rectangular & 169.95 \\
\hline L700Ja1b100h40 & Elliptical & 171.27 \\
\hline L700Ja1b100h60 & Rectangular & 162.56 \\
\hline L700Ja1b100h60 & Elliptical & 163.28 \\
\hline L700Jb1b100h40 & Rectangular & 180.29 \\
\hline L700Jb1b100h40 & Elliptical & 181.69 \\
\hline L700Jb1b100h60 & Rectangular & 174.14 \\
\hline L700Jb1b100h60 & Elliptical & 173.37 \\
\hline
\end{tabular}


Table 4 Bearing Capacity Of Perforated Members With Different Hole Spacing

\begin{tabular}{|c|c|c|}
\hline Specimen & $l / \mathrm{mm}$ & $P_{\mathrm{u}} / \mathrm{kN}$ \\
\hline L1500Ja6b100h40 & 600 & 100.19 \\
\hline L1500Ja6b100h40 & 700 & 96.99 \\
\hline L1500Ja6b100h40 & 800 & 99.09 \\
\hline L1500Jb4b100h40 & 600 & 109.66 \\
\hline L1500Jb4b100h40 & 700 & 111.32 \\
\hline L1500Jb4b100h40 & 800 & 115.49 \\
\hline L1500Jb12b100h40 & 600 & 125.36 \\
\hline L1500Jb12b100h40 & 700 & 123.53 \\
\hline L1500Jb12b100h40 & 800 & 128.40 \\
\hline
\end{tabular}

Table 5 Bearing Capacity Of Perforated Lipped Channel With Different Hole Heights

\begin{tabular}{|c|c|c|}
\hline Specimen & $P_{\mathrm{u}} / \mathrm{kN}$ & $\begin{array}{c}\text { Decreased } \\
\text { percentage }\end{array}$ \\
\hline L700Jb1b110h20 & 183.47 & 0.97 \\
\hline L700Jb1b110h30 & 181.21 & 2.19 \\
\hline L700Jb1b110h40 & 177.99 & 3.92 \\
\hline L700Jb1b110h50 & 175.44 & 5.30 \\
\hline L700Jb1b110h60 & 172.79 & 6.73 \\
\hline L700Jb1b110h70 & 167.81 & 9.42 \\
\hline L700Jb1b110h80 & 162.87 & 12.09 \\
\hline L700Jb1b110h100 & 154.40 & 16.66 \\
\hline L700Jb2b110h20 & 198.63 & 0.80 \\
\hline L700Jb2b110h30 & 197.34 & 1.45 \\
\hline L700Jb2b110h40 & 195.02 & 2.61 \\
\hline L700Jb2b110h50 & 191.45 & 4.39 \\
\hline L700Jb2b110h60 & 187.94 & 6.14 \\
\hline L700Jb2b110h70 & 184.37 & 7.93 \\
\hline L700Jb2b110h80 & 180.78 & 9.72 \\
\hline L700Jb2b110h100 & 176.29 & 11.96 \\
\hline L1600Jb1b110h20 & 171.77 & 0.60 \\
\hline L1600Jb1b110h30 & 170.55 & 1.30 \\
\hline L1600Jb1b110h40 & 168.14 & 2.70 \\
\hline L1600Jb1b110h50 & 165.33 & 4.32 \\
\hline L1600Jb1b110h60 & 162.63 & 5.89 \\
\hline L1600Jb1b110h70 & 159.86 & 7.49 \\
\hline L1600Jb1b110h80 & 157.11 & 9.08 \\
\hline L1600Jb1b110h100 & 147.78 & 14.48 \\
\hline L1600Jb3b110h20 & 302.19 & 1.02 \\
\hline L1600Jb3b110h30 & 296.17 & 2.99 \\
\hline L1600Jb3b110h40 & 292.81 & 4.09 \\
\hline
\end{tabular}




\begin{tabular}{|c|c|c|}
\hline L1600Jb3b110h50 & 288.13 & 5.62 \\
\hline L1600Jb3b110h60 & 283.22 & 7.23 \\
\hline L1600Jb3b110h70 & 279.05 & 8.60 \\
\hline L1600Jb3b110h80 & 274.43 & 10.11 \\
\hline L1600Jb3b110h100 & 267.65 & 12.33 \\
\hline
\end{tabular}

Note: decreased percentage means compared with mem bers with no hole.

\section{The Direct Strength Method for Perforated Lipped Channel Columns}

\subsection{Elastic buckling stress of axial compression members}

Based on a large number of finite strip analysis, the simplified calculation method of elastic critical stress of lipped perforated channels are presented by literature [2]. To maintain the local elastic buckling stress and global buckling stress of the members same as members with no hole. And for the elastic distortion buckling stress, actual thickness of the web is replaced by discounting web thickness. The following is reduction method. The contrast between the results of finite element analysis and the simplified method is listed in Table 6. And the simplified method is validated.

$$
t_{\mathrm{web}, \mathrm{hole}}=\left(1-\frac{b}{L}\right) t_{\mathrm{web}}
$$

Where $b$ means the width of hole, $L$ means the length of specimen, $t_{\text {web }}$ means actual web thickness.

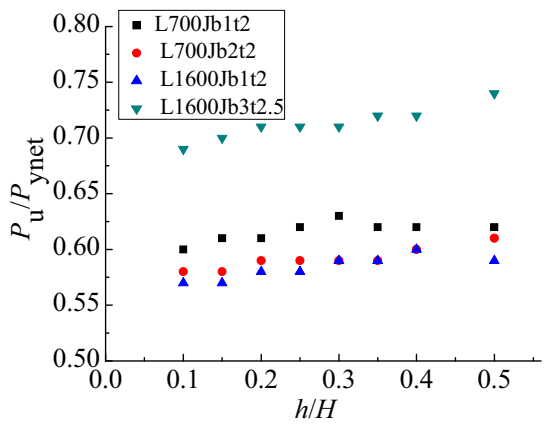

Fig. 3 Relationship of $P_{\mathrm{u}} / P_{\text {ynet }}$ and $h / H$

Table 6 Distortional Buckling Stress Calculation Results By Simplified Thickness Reduction Method

\begin{tabular}{|c|c|c|}
\hline Specimen & $\begin{array}{c}\text { Calculation } \\
\text { results } \\
\sigma_{\mathrm{cr}, \mathrm{cal}} / \mathrm{MPa}\end{array}$ & $\begin{array}{c}\text { Simulation results } \\
\sigma_{\mathrm{cr}, \text { sim }} / \mathrm{MPa}\end{array}$ \\
\hline L900Ja10b80h60 & 79.50 & 86.41 \\
\hline L900Ja10b100h60 & 79.50 & 86.47 \\
\hline L800Ja5b100h40 & 313.79 & 339.40 \\
\hline L700Jb1b40h40 & 102.66 & 116.05 \\
\hline L1200Jb10b110h40 & 191.72 & 177.37 \\
\hline
\end{tabular}




\subsection{The DSM for lipped perforated channels under axial compression}

Moen had put forward a reasonable method for lipped perforated channels under axial compression [3]. The method not only considers the fact that nominal capacity for local-global interaction buckling is not more than the net section bearing capacity, but also is more detailed to segment the calculation formula of distortion.

Referred to the method above, combined with the domestic modified DSM for members with no holes, the domestic DSM for lipped perforated channels under axial compression is proposed. The formula is as follows.

The proposed nominal axial capacity for the interaction of local-overall buckling $P_{\mathrm{nl}}$ is as follows:

$$
P_{\mathrm{n} l}=\left\{\begin{array}{cc}
P_{\mathrm{ne}} & \lambda_{l} \leq 0.847 \\
{\left[1-0.10\left(\frac{P_{\mathrm{cr} l}}{P_{\mathrm{ne}}}\right)^{0.36}\right]\left(\frac{P_{\mathrm{cr} l}}{P_{\mathrm{ne}}}\right)^{0.36} N_{\mathrm{ne}}} & \lambda_{l}>0.847
\end{array}\right.
$$

And $P_{\mathrm{n} l} \leq P_{\text {ynet }}$

The proposed nominal capacity for the interaction of distortional-overall buckling $P_{\mathrm{nd}}$ is as follows:

$$
P_{\text {nd }}=\left\{\begin{array}{cc}
P_{\text {ynet }} & \lambda_{\mathrm{d}} \leq \lambda_{\mathrm{d} 1} \\
P_{\mathrm{ynet}}-\left(\frac{P_{\mathrm{ynet}}-P_{\mathrm{d} 2}}{\lambda_{\mathrm{d} 2}-\lambda_{\mathrm{d} 1}}\right)\left(\lambda_{\mathrm{d}}-\lambda_{\mathrm{d} 1}\right) & \lambda_{\mathrm{d} 1}<\lambda_{\mathrm{d}} \leq \lambda_{\mathrm{d} 2} \\
{\left[1-0.25\left(\frac{P_{\mathrm{crd}}}{P_{\mathrm{ne}}}\right)^{0.6}\right]\left(\frac{P_{\mathrm{crd}}}{P_{\mathrm{ne}}}\right)^{0.6} P_{\mathrm{ne}}} & \lambda_{\mathrm{d} 2}<\lambda_{\mathrm{d}}
\end{array}\right.
$$

Where $\lambda_{\mathrm{d} 1}=0.561\left(P_{\text {ynet }} / P_{\text {ne }}\right)$

$$
\begin{aligned}
& \lambda_{\mathrm{d} 2}=0.561\left[14\left(P_{\mathrm{ne}} / P_{\mathrm{ynet}}\right)^{0.4}-13\right] \\
& P_{d 2}=\left(1-0.25\left(\frac{1}{l_{d 2}}\right)^{1.2}\right)\left(\frac{1}{l_{d 2}}\right)^{1.2} P_{n e}
\end{aligned}
$$

The ultimate bearing capacity of the mentioned specimen above is calculated by the formula proposed. The comparison between the results and the simulation results are shown in Table 7. The other results were found in the literature [4].The contrast results are in good agreement. 


\subsection{The Direct Strength Method for Complex Lipped Perforated Channel Columns}

The proposed method above is suited for lipped perforated channel columns. In order to study the DSM for complex lipped perforated channel columns, the ultimate bearing capacities of complex lipped perforated channels were calculated using the method above and compared with the results of finite element simulation. As shown in Table 8. The method for lipped perforated channels to solve the ultimate bearing capacity of complex lipped perforated channel is still applicable.

Table 7 Results Of Lipped Perforated Channels Under Axial Compression By Dsm

\begin{tabular}{|c|c|c|c|c|}
\hline Specimen & $P_{\mathrm{E}} / \mathrm{kN}$ & $P_{\mathrm{D}} / \mathrm{kN}$ & $\begin{array}{c}\text { Buckling } \\
\text { modes }\end{array}$ & $P_{\mathrm{D}} / P_{\mathrm{E}}$ \\
\hline L700Ja7b40h40 & 165.48 & 153.99 & $\mathrm{D}$ & 0.93 \\
\hline L700Ja7b70h40 & 164.47 & 149.96 & $\mathrm{D}$ & 0.91 \\
\hline L700Ja7b100h40 & 163.07 & 145.82 & $\mathrm{D}$ & 0.89 \\
\hline L700Ja1b40h40 & 169.55 & 169.94 & $\mathrm{D}$ & 1.00 \\
\hline L700Ja1b70h40 & 170.82 & 167.93 & $\mathrm{D}$ & 0.98 \\
\hline L700Ja1b100h40 & 169.95 & 163.42 & $\mathrm{D}$ & 0.96 \\
\hline L1600Ja1b110h20 & 162.56 & 158.02 & $\mathrm{D}$ & 0.97 \\
\hline L1600Ja1b110h30 & 161.02 & 158.02 & $\mathrm{D}$ & 0.98 \\
\hline L1600Ja1b110h40 & 159.48 & 158.02 & $\mathrm{~L}$ & 0.99 \\
\hline L1600Ja1b110h50 & 156.83 & 158.02 & $\mathrm{D}$ & 1.01 \\
\hline L1600Ja1b110h60 & 154.24 & 158.02 & $\mathrm{D}$ & 1.02 \\
\hline L1600Ja1b110h70 & 151.52 & 158.02 & $\mathrm{D}$ & 1.04 \\
\hline L1600Ja1b110h80 & 148.80 & 158.02 & $\mathrm{D}$ & 1.06 \\
\hline L1600Ja1b110h100 & 135.26 & 158.02 & $\mathrm{D}$ & 1.17 \\
\hline Average & - & - & - & 0.994 \\
\hline StDev & - & - & - & 0.069 \\
\hline
\end{tabular}

Notes: L means local buckling, D means distortional buckling.

Table 8 Results Of Complex Lipped Perforated Channels Under Axial Compression By Dsm

\begin{tabular}{|c|c|c|c|c|}
\hline Specimen & $P_{\mathrm{E}} / \mathrm{kN}$ & $P_{\mathrm{D}} / \mathrm{kN}$ & $\begin{array}{c}\text { Buckling } \\
\text { modes }\end{array}$ & $P_{\mathrm{D}} / P_{\mathrm{E}}$ \\
\hline L700Jb1b40h40 & 181.46 & 184.43 & $\mathrm{~L}$ & 1.02 \\
\hline L700Jb1b40h70 & 181.59 & 184.43 & $\mathrm{~L}$ & 1.02 \\
\hline L700Jb1b40h100 & 180.29 & 184.43 & $\mathrm{~L}$ & 1.02 \\
\hline L1600Jb1b110h20 & 171.77 & 172.5 & $\mathrm{~L}$ & 1.00 \\
\hline L1600Jb1b110h30 & 170.55 & 172.5 & $\mathrm{~L}$ & 1.01 \\
\hline L1600Jb1b110h40 & 168.14 & 172.5 & $\mathrm{~L}$ & 1.03 \\
\hline L1200Jb8b110h40 & 181.53 & 182.68 & $\mathrm{D}$ & 1.01 \\
\hline L1200Jb8b110h60 & 170.18 & 182.68 & $\mathrm{D}$ & 1.07 \\
\hline L1200Jb8b110h80 & 165.36 & 182.68 & $\mathrm{D}$ & 1.10 \\
\hline L1200Jb9b110h40 & 357.74 & 340.19 & $\mathrm{D}$ & 0.95 \\
\hline L1200Jb9b110h60 & 327.92 & 340.19 & $\mathrm{D}$ & 1.04 \\
\hline
\end{tabular}




\begin{tabular}{|c|c|c|c|c|}
\hline L1200Jb9b110h80 & 316.52 & 340.19 & $\mathrm{D}$ & 1.07 \\
\hline L1200Jb10b110h30 & 174.16 & 160.06 & $\mathrm{D}$ & 0.92 \\
\hline L1200Jb10b110h40 & 164.51 & 160.06 & $\mathrm{D}$ & 0.97 \\
\hline L1200Jb10b110h50 & 159.55 & 160.06 & $\mathrm{D}$ & 1.00 \\
\hline Average & - & - & - & 1.015 \\
\hline StDev & - & - & - & 0.046 \\
\hline
\end{tabular}

\section{Summary}

(1) The influence of the width of hole, hole shape and hole spacing on the buckling mode of members is very small. It is suggested that the hole width of the cold-formed channel is $110 \mathrm{~mm}$.

(2) The influence of the hole height on the stability of the bearing capacity is large. Hole height should be 30-40 percent of the height of web.

(3) Based on the domestic modified DSM, a method for lipped perforated channels under axial compression is proposed and verified by the finite element analysis results. The method is also suited for complex lipped perforated channels.

\section{Acknowledgements}

This work described in this paper was supported by Natural Science Foundation of Liaoning Province (2015020575), their supports are gratefully acknowledged.

\section{References}

1. Wang Chungang, Zhang Zhuangnan, Zhao Daqian, Cao Yufei. Experimental investigation of $\Sigma$ - section channel steel with complex edge stiffeners and web holes under axial compression. Journal of Jilin University (Engineering and Technology Edition). 2015, 45 (3):788-796.

2. Sputo T, Tovar J. Application of direct strength method to axially loaded perforated cold-formed steel studs: Longwave buckling [J]. Thin-Walled Structures, 2005, 43(12): 1852-1881.

3. Moen C D, Schafer B W. Direct strength method for design of cold-formed steel columns with holes[J]. Journal of Structural Engineering, 2011, 137(5): 559-570.

4. Zhao Daqian. Research on Direct Strength Method of Perforated Lipped Channel Axial Compression Bearing Capacity [D]. Shenyang, Shenyang Jianzhu University. 2014 